\title{
THERMAL MODEL OF AN AIRSHIP WITH SOLAR ARRAYS AND A
}

\section{BALLONET}

\author{
D. Greenhalgh ${ }^{1}$, A. R. Tatnall ${ }^{2}$ \\ ${ }^{1}$ Faculty of Engineering and the Environment, University of Southampton, UK \\ ${ }^{2}$ Faculty of Engineering and the Environment, University of Southampton, UK
}

\begin{abstract}
Airships and other lighter than air vehicles depend on a lifting gas to provide buoyancy. This lifting gas is subject to changes in temperature due to external heating which can affect its pressure and volume. Many airship designs contain ballonet systems to allow the lifting gas to maintain a set pressure despite changes in temperature. This study improves the accuracy of airship thermal models by incorporating the effects of a ballonet system. A transient thermal model based on a finite element discretisation for the structure and lumped averages for the gases was developed to account for the effects of a ballonet system by formulating the first law of thermodynamics in conjunction with an ideal gas representation of the lifting gas and ballonet air. Solutions to the resulting differential equation were found via the implicit Euler method using a Newton-Krylov solver. The results of the simulation were compared to previously published experimental results of an airship with a solar array and ballonet systems. The simulation results show a good agreement with the experimental results $(r \geq 0.9)$. As such, this model can be used to aid in the design of future airships and aerostats with ballonet structures.
\end{abstract}

Keywords: Airship, Aerostat, Thermal Analysis, Balloonet

\section{INTRODUCTION}

Aerostats and airships are lighter-than-air aircraft which have received renewed interest in recent years for high altitude applications including surveillance, communications and power-raising. A power-raising aerostat was proposed by Aglietti et al. [1-4] and Redi et al. [5,6] and consists of a 65 $\mathrm{m}$ diameter spherical aerostat with a $2500 \mathrm{~m}^{2}$ solar array. The aerostat would float at approximately $6 \mathrm{~km}$ altitude where, if tethered in southern England, it can receive over three times the annual energy of a ground based system [5]. The electrical power will be transmitted to the ground via the tether. The aerostat will need to keep a constant shape during its ascent and operation despite changes in atmospheric pressure; as such a ballonet will be used to maintain a constant pressure inside the aerostat. The temperature of the lifting gas will directly affect its pressure and the pressure differential between the contained gas and the external atmosphere defines the stress experienced by the aerostat envelope. In balloons, which are allowed to expand and contract or aerostats and airships with ballonet systems in which the lifting gas can also expand or contract, the lifting gas temperature will also influence the buoyancy of the system. Thermal analysis can therefore be used to assess the feasibility of an aerostat or airship design.

Much work has been done on the thermal analysis of scientific balloons to aid in the design and flight profile prediction [7-11]. Scientific balloons are typically made from flexible films and pressurised such that they expand until they reach their float altitude at which they are fully expanded and the gas pressure is equal to the ambient air pressure. Airships and aerostats are instead required to maintain their shape during ascent and as such are designed with ballonets which allow air to enter or exit the envelope in order to maintain the internal super-pressure in the face of changing atmospheric pressure. Although some work has been done to develop thermal models of airships and aerostats [12-15], some of which include the effects of affixed solar arrays $[16,17]$, no work has been done to model the effects of a ballonet system used to control the internal gas pressure as the temperature changes.

This paper describes a new thermal model for an aerostat or airship that builds on previously published models but extends them to include the effects of a ballonet used for gas pressure control. The model is then validated against experimental results of an airship.

\section{THERMAL MODEL}

\subsection{Model Discretisation}

The airship model consists of five parts: the solar array, the envelope, the lifting gas, the ballonet membrane and the ballonet air and is illustrated in Fig. 1. The envelope and ballonet membrane are modelled as single layered membranes and discretised into triangular elements. The solar array is modelled as three layers stacked upon each other: the cover glass, the photovoltaic cells and the substrate to which these are mounted. The solar array layers are also discretised into triangular elements. The lifting gas and ballonet air are treated as lumped averages. 


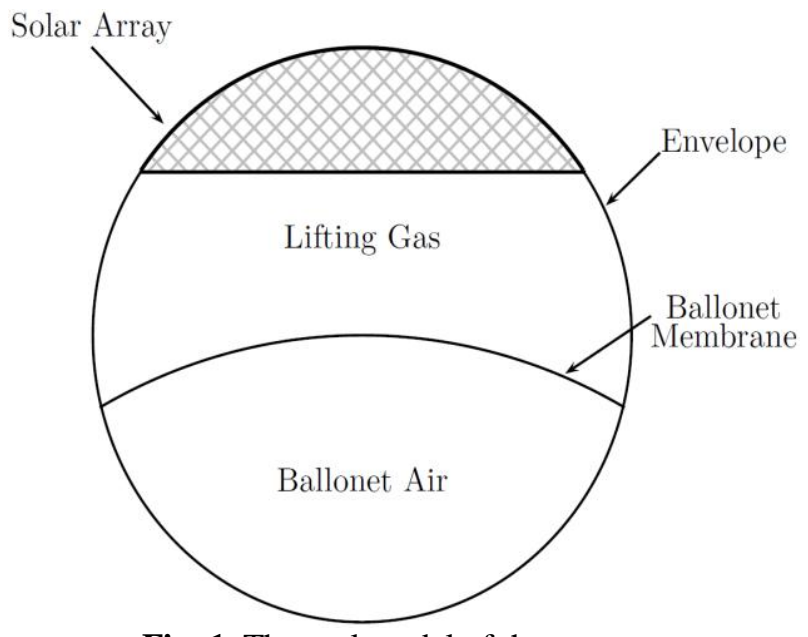

Fig. 1. Thermal model of the aerostat

\subsection{Governing Equations}

The heat transfer of the airship system is modelled using the first law of thermodynamics:

$$
\dot{U}=\dot{Q}-\dot{W}
$$

Where $U$ is the internal energy, $Q$ is the applied heat load and $W$ is the work done by the system.

\subsection{Internal Energy}

The internal energy for any element $i$ can be described as

$$
U_{i}=c_{i} m_{i} T_{i}
$$

Where $c_{i}$ is the specific heat capacity of the element, $m_{i}$ is the element's mass and $T_{i}$ its temperature, thus

$$
\dot{U}_{l}=\frac{d}{d t}\left(c_{i} m_{i} T_{i}\right)
$$

For the solid elements, i.e. the envelope, the solar array and the ballonet membrane the mass is constant so equation (3) can be simplified to

$$
\dot{U}_{i}=c_{i} m_{i} \frac{d T_{i}}{d t}
$$

For the lifting gas, the mass is also constant as no mass flow is allowed in or out of the lifting gas section. The specific heat capacity used is that at constant volume, so equation (3) becomes

$$
\dot{U}_{g}=c_{v, g} m_{g} \frac{d T_{g}}{d t}
$$

For the ballonet air the mass is not constant as it can flow in and out of the ballonet as the lifting gas pressure varies. Therefore equation (3) becomes

$$
\dot{U}_{b}=\dot{m}_{b} c_{v, b} T_{b}+c_{v, b} m_{b} \frac{d T_{b}}{d t}
$$

\subsection{Thermal Loads}

\subsubsection{Envelope}

The rate of heat transfer to the envelope is

$$
\sum \dot{Q}_{n}=\dot{Q}_{S}+\dot{Q}_{\mathrm{IR}}-\dot{Q}_{\mathrm{CE}}-\dot{Q}_{\mathrm{CI}}+\dot{Q}_{\mathrm{INT}}+\dot{Q}_{\mathrm{subs}, \mathrm{env}}
$$

where $\dot{Q}_{S}$ is the absorbed solar radiation incorporating direct, diffuse and reflected radiation, $\dot{Q}_{\mathrm{IR}}$ is the rate of heat change due to absorbed and emitted infrared radiation, $\dot{Q}_{\mathrm{CE}}$ is the rate of heat transfer due to external convection, $\dot{Q}_{\mathrm{CI}}$ is the rate of heat transfer due to internal convection, $\dot{Q}_{\mathrm{INT}}$ is the internally emitted and absorbed infrared radiation, also known as self glow [9] and $\dot{Q}_{\text {subs,env }}$ is the rate of conduction from the solar array substrate to the envelope.

\subsubsection{Solar Array}

The solar array model uses the three layer model used by $\mathrm{Li}$ et al [16] of a cover glass, a photovoltaic cell array and a substrate. This model is shown in Fig. 2.

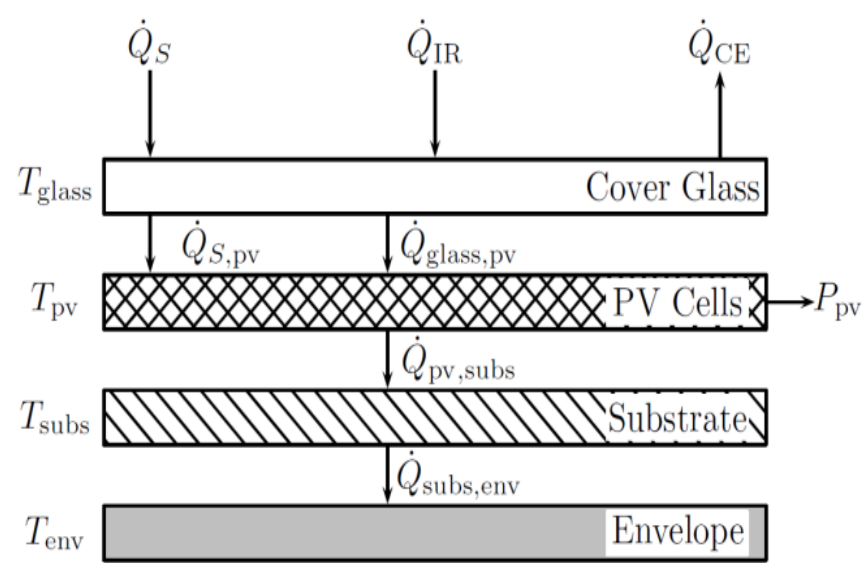

Fig. 2. Solar array model [16]

The rates of heat transfer through the solar array layers are thus

$$
\begin{gathered}
\sum \dot{Q}_{n, \mathrm{glass}}=\dot{Q}_{S}+\dot{Q}_{\mathrm{IR}}-\dot{Q}_{\mathrm{CE}}-\dot{Q}_{\mathrm{glass}, \mathrm{pv}} \\
\sum \dot{Q}_{n, \mathrm{pv}}=\dot{Q}_{S, \mathrm{pv}}+\dot{Q}_{\mathrm{glass}, \mathrm{pv}}-\dot{Q}_{\mathrm{pv}, \mathrm{subs}} \\
\sum \dot{Q}_{n, \text { subs }}=\dot{Q}_{\mathrm{pv}, \mathrm{subs}}+\dot{Q}_{\mathrm{subs}, \mathrm{env}}
\end{gathered}
$$

where $\dot{Q}_{S}, \dot{Q}_{\mathrm{IR}}$ and $\dot{Q}_{\mathrm{CE}}$ are as above, $\dot{Q}_{S, \mathrm{pv}}$ is the solar radiation that falls on the photovoltaic cells (i.e. $\dot{Q}_{S}$ attenuated by the cover glass), and $\dot{Q}_{\text {glass,pv }}, \dot{Q}_{\mathrm{pv}, \text { subs }}$ and $\dot{Q}_{\text {subs,env }}$ are the heat conduction between the layers of the solar array. Glass is opaque to infra-red radiation and it is assumed that all the 
ground and sky IR radiation that is not absorbed by the glass is reflected. Therefore there is no attenuated IR radiation falling on the photovoltaic cells.

\subsubsection{Ballonet Membrane}

As shown in Fig. 1 the membrane splits the internal volume of the airship into two parts. The upper surface of the membrane will be subject to radiative heat transfer with the upper elements of the envelope internal surface and convective heat transfer with the lifting gas. The lower surface of the membrane will exchange radiation with the lower elements of the envelope internal surface and have convection with the ballonet air. These processes are described in Sections 2.4.4, 2.4.5 and 2.4.10.

\subsubsection{Lifting Gas}

The lifting gas will exchange heat with the airship envelope and the top surface of the ballonet membrane via convection: the heat flux to the lifting gas is the sum of the internal convection terms of all elements of the ballonet membrane on the lifting gas side and all envelope elements above the ballonet membrane.

$$
\dot{Q}_{\mathrm{gas}}=\sum_{i} \dot{Q}_{\mathrm{Cl}, i} \forall i \in\left(\mathbf{E}_{t} \cup \mathbf{M}\right)
$$

where $\dot{Q}_{\text {gas }}$ is the heat flux to the lifting gas, $\dot{Q}_{\mathrm{CI}, i}$ is the internal convective heat flux from element $i, \mathbf{E}_{t}$ is the set of envelope elements above the membrane and $\mathbf{M}$ is the set of membrane elements.

\subsubsection{Ballonet Air}

Like the lifting gas, the ballonet air will exchange heat with its surroundings: the lower portion of the envelope and the lower surface of the membrane:

$$
\dot{Q}_{b}=\sum_{i} \dot{Q}_{\mathrm{Cl}, i} \forall i \in\left(\mathbf{E}_{b} \cup \mathbf{M}\right)
$$

where $\dot{Q}_{b}$ is the heat flux to the ballonet air, $\mathbf{E}_{b}$ is the set of envelope elements below the membrane.

\subsubsection{Solar Radiation}

There are three sources of solar radiation that will cause thermal loads on the airship: direct radiation from the Sun, diffuse radiation from direct radiation which has been scattered by the atmosphere and radiation reflected from the Earth's surface. These are designated by $I_{D}, I_{S}$ and $I_{R}$ respectively and are calculated, along with the solar angle using a modified version of the SMARTS2 program [18]. The incident radiation on an element will be the sum of the three of these multiplied by their respective incidence angles. For direct beam radiation, the incident radiation is

$$
R_{D, i}=A_{i} I_{D} \cos \left(\theta_{i}\right)
$$

where $A_{i}$ is the element area and $\theta_{i}$ is the angle of incidence. If the angle of incidence is outside the range $\left[-90^{\circ}, 90^{\circ}\right]$ then $\cos \left(\theta_{i}\right)=0$. The incident diffuse radiation on an element will be [11]

$$
R_{S, i}=A_{i} I_{S}\left(\frac{1-\cos \left(\phi_{i}\right)}{2}\right)
$$

where $\phi_{i}$ is the angle from the element normal to the ground. Likewise, if $\phi_{i}$ is outside the range $\left[-90^{\circ}, 90^{\circ}\right]$ then $\cos \left(\phi_{i}\right)=0$. The reflected incident radiation will be [11]

$$
R_{R, i}=A_{i} I_{R}\left(\frac{1+\cos \left(\phi_{i}\right)}{2}\right)
$$

The absorbed incident solar radiation on an element will be

$$
\dot{Q}_{S, i}=\alpha_{i}\left(R_{D, i}+R_{S, i}+R_{D, i}\right)
$$

where $\alpha_{i}$ is the absorptivity of the element. Only the elements of the envelope that are not covered by the solar array will receive solar radiation along with all elements of the cover-glass. The radiation the solar cells receive will have been attenuated by the cover glass and so the absorbed incident radiation on an element of the solar cells will be

$$
\dot{Q}_{S, i}=\left(1-\alpha_{\text {glass }}\right) \alpha_{\mathrm{pv}}\left(R_{D, i}+R_{S, i}+R_{D, i}\right)
$$

where $\alpha_{\mathrm{pv}}$ is the absorptivity of the solar cells. Some of this absorbed radiation will be carried away in the form of electrical power as discussed in Section 2.5.1.

\subsubsection{Infrared Radiation}

The airship will absorb infrared radiation from the ground and from the sky and emit infrared radiation. The envelope and solar arrays cover glass are assumed to be grey bodies where the absorptivity in the infrared range is equal to their emissivities. The rate of change of heat due to infra-red radiation is

$$
\dot{Q}_{\mathrm{IR}, i}=\varepsilon_{i} A_{i}\left(q_{g} \Phi_{i}+q_{\mathrm{sky}}\left(1-\Phi_{i}\right)-\sigma T_{i}^{4}\right)
$$

Where $\sigma$ is the Stefan-Boltzmann constant, $\varepsilon_{i}$ is the element's emissivity, $T_{i}$ is the element's temperature, $q_{g}$ is the infra-red radiation from the ground at the airship and $q_{\text {sky }}$ that from the sky. $\Phi_{i}$ is the view factor of the Earth from the element, calculated using the method in Louchev (1992) [19]. In this model, the radiation from the ground and sky are given in the experimental results (see Section 3), however they can be calculated as

$$
q_{g}=\sigma \tau \varepsilon_{g} T_{g}^{4}
$$

And

$$
q_{s k y}=\sigma T_{s k y}^{4}
$$


Where $\varepsilon_{g}$ is the emissivity of the ground, values for which can be found in [20], $T_{g}$ is the ground temperature, $\tau$ is the transmissivity of the atmosphere to IR radiation, given in [9] and $T_{s k y}$ is the effective sky temperature, given in [11].

\subsubsection{Internal Radiation}

The envelope will radiate internally as well as externally. Helium is transparent to infrared radiation [7] and it is assumed that the air in the ballonet is also, so all the internally emitted radiation must be absorbed or reflected by the envelope. The heat transfer from the internal radiation is

$$
\dot{Q}_{\mathrm{INT}, i}=A_{i}\left(G_{i}-H_{i}\right)
$$

where $G_{i}$ and $H_{i}$ are the infrared radiation absorbed by and emitted by element $i$ respectively:

$$
H_{i}=\varepsilon_{\mathrm{INT}} \sigma T_{i}^{4}
$$

And

$$
G_{i}=\sum_{k=1}^{N} J_{k} \phi_{i, k}
$$

where $\varepsilon_{\mathrm{INT}}$ is the emissivity of the internal surface of the envelope, $\phi_{i, k}$ is the view factor from element $i$ to element $k$ calculated using the program view3d [21] and $N$ is the number of envelope elements in the mesh. There are two meshes used for internal radiation calculations in this model, one for the lifting gas section that includes the envelope elements above the membrane and the membrane elements and the other for the ballonet section which contains all the envelope elements below the membrane and the membrane elements. $J_{k}$ is the sum of the infrared radiation emitted by element $k$ and the incident radiation from other elements which is reflected by element $k$ :

$$
J_{k}=H_{k}+\left(1-\varepsilon_{\mathrm{INT}}\right) \sum_{l=1}^{N} J_{l} \phi_{k, l}
$$

Thus the term for $J_{k}$ contains all other $J$ terms to account for the internal reflection of radiation within the airship. Li et al [16] showed that equation (24) can be transformed into the form

$$
\left[\begin{array}{ccc}
a_{1,1} & \cdots & a_{1, N} \\
\vdots & \ddots & \vdots \\
a_{N, 1} & \cdots & a_{N, N}
\end{array}\right] \cdot\left[\begin{array}{c}
J_{1} \\
\vdots \\
J_{N}
\end{array}\right]=\sigma\left[\begin{array}{c}
T_{1}^{4} \\
\vdots \\
T_{N}^{4}
\end{array}\right]
$$

Where

$$
\alpha_{i, k}= \begin{cases}\frac{1}{\varepsilon_{\mathrm{INT}}}, & i=k \\ \frac{\left(\varepsilon_{\mathrm{INT}}-1\right)}{\varepsilon_{\mathrm{INT}}}, & i \neq k\end{cases}
$$

and $G_{i}$ can be re-written in terms of $J_{i}$ :

$$
G_{i}=\frac{J_{i}-H_{i}}{1-\varepsilon_{\mathrm{INT}}}
$$

Equation (25) is solved twice, once for the lifting gas section and once for the ballonet section.

In reality, the membrane will move as the lifting gas and ballonet air change volume and this will change the view factors between all the elements. However, calculating the view factor matrices $a$, is the most computationally expensive step of the simulation. Therefore, the view factor matrices are calculated only once at the start of the simulation with the membrane assumed to be flat. These matrices are then used throughout the simulation. Because the individual view factors are very small and the internal radiation terms only redistributes heat around the envelope and membrane and does not represent heat added to or lost by the system as a whole it is thought that this will result only in a small error.

\subsubsection{External Convection}

The rate of heat transfer due to convection for an element of the airship surface (either cover glass or envelope), $\dot{Q}_{\mathrm{CE}, i}$, will be

$$
\dot{Q}_{C E, i}=A_{i} \frac{k_{\mathrm{air}}}{L} N u\left(T_{i}-T_{\mathrm{air}}\right)
$$

where $k_{\text {air }}$ is the thermal conductivity of air, $L$ is the characteristic length, which is taken as the mean of the airship's length and diameter and the Nusselt number, $N u$, for an airship is given by

$$
N u=\left\{\begin{array}{cc}
2+0.47 \sqrt{R e_{\text {air }}} P r_{\text {air' }}^{\frac{1}{3}} & R e_{\text {air }} \leq 5 \times 10^{5} \\
\left(0.0262 R e_{\text {air }}^{0.8}-615\right) P r_{\text {air' }}^{\frac{1}{3}} & R e_{\text {air }}>5 \times 10^{5}
\end{array}\right.
$$

where $R e_{\text {air }}$ is the Reynold's number and $P r_{\text {air }}$ is the Prandtl number of air.

\subsubsection{Internal Convection}

The convective behaviour inside the lifting gas is a complex problem and one that will not be addressed here. Instead, only the heat transfer from an envelope element to the lifting gas is considered. The rate of this heat transfer, $\dot{Q}_{\mathrm{Cl}, i}$, can be modelled as [7]

$$
\dot{Q}_{C I, i}=0.13 \frac{k_{\text {gas }}}{L} G r_{\text {gas }}^{\frac{1}{3}} A_{i}\left(T_{i}-T_{\text {gas }}\right)
$$

where $k_{\text {gas }}$ and $G r_{\text {gas }}$ are the thermal conductivity and the Grashoff number of the lifting gas.

\subsubsection{Conduction}

In the solar array there will be conduction from one layer to another although conduction between elements of each layer 
is ignored in this analysis. The heat transfer between one layer and the adjacent layer is

$$
\dot{Q}_{i, i+1}=A_{i}\left(T_{i}-T_{i+1}\right) /\left(\frac{d_{i}}{k_{i}}+\frac{d_{i+1}}{k_{i+1}}\right)
$$

where $d_{i}$ and $k_{i}$ are the thickness and thermal conductivity of layer $i$ respectively. Conduction from the solar array substrate to the airship hull is considered but conduction from the outer layer to the inner layer of the envelope is not. This is because the envelope material is very thin so the temperature can be assumed to be constant throughout its cross-section. Additionally, no conduction over the surface of the envelope is considered due to the fact that each element will have a temperature very close to its neighbours and so the heat flux by conduction between elements will be very small in comparison to the heat fluxes already described. Previous studies $[11,16]$ use this approach to reduce the complexity (and thus computational time) of the simulation without much loss of accuracy.

\subsection{Work Done}

\subsubsection{Solar Array}

The solar array will do work in the form of generating electrical power:

$$
\dot{W}_{\mathrm{pv}, i}=P_{\mathrm{pv}, i=} \dot{Q}_{S, \mathrm{pv}, i} \eta_{0}\left[1-c_{-} t\left(T i-T_{0}\right)\right]
$$

where $P_{\mathrm{pv}, i}$ is the generated electrical power, $\eta_{0}$ is the efficiency at a temperature $T_{0}$ and $c_{t}$ is the efficiencytemperature coefficient, measured in $\mathrm{K}^{-1}$. In this model, $\eta_{0}=0.2$ at standard test conditions, that is a temperature of $298.15 \mathrm{~K}\left(25^{\circ} \mathrm{C}\right)$ and $c_{t}=10^{-3} \mathrm{~K}^{-1}$.

\subsubsection{Lifting Gas}

The lifting gas will do pressure-volume work as its volume changes:

$$
\dot{W}_{g}=p_{g} \frac{d V_{g}}{d t}
$$

\subsubsection{Ballonet Air}

The ballonet air will also do pressure-volume work as its volume changes and will do flow work as air flows in and out of the ballonet:

$$
\dot{W}_{b}=p_{b} \frac{d V_{b}}{d t}-c_{p} \dot{m}_{\mathrm{in}} T_{\mathrm{amb}}+c_{p} \dot{m}_{\mathrm{out}} T
$$

The mass flow into and out of the ballonet can be modelled as flow through a valve [22]. The mass flow rate, $\dot{m}$, through the valve is

$$
\dot{m}=\mu A_{v} \sqrt{2 \rho|\Delta p|} \cdot \operatorname{sign}(\Delta p)
$$

where $\mu$ is the discharge coefficient, $A_{v}$ is the cross-sectional area of the valve, $\Delta p$ is the pressure difference between the air in the ballonet and the external ambient air and $\rho$ is the air density. For positive $\Delta p$ air flows into the ballonet and $\rho$ is for the ambient air, for negative $\Delta p$ air flows out of the ballonet and $\rho$ is for the air inside the ballonet. The density of air inside and outside the ballonet will be different because the air in the ballonet will be maintained at a higher pressure than atmospheric and may have a different temperature to the ambient air because of convective heating from the envelope and membrane. In reality maintaining a super-pressure would require a control system capable of sensing the pressure difference between the ballonet and external air and using a fan system to bring in air from the atmosphere if the pressure fell below the desired super-pressure and a release valve to expel air when the pressure increases above the desired super-pressure. In this model, the super-pressure is accounted for in $\Delta p$ so the simple valve model can be used and the control aspect neglected.

\subsection{Solution Method}

\subsubsection{Numerical Method}

Substituting equations (2), (3), (4), (32) and (33) into equation (1) and re-arranging gives the rate of change of temperature:

$$
\frac{d \mathbf{T}}{d t}=\left[c_{v} m\right]^{-1}\left[\dot{\mathbf{Q}}+R\left(\dot{\mathbf{m}}_{\mathrm{in}} T_{\mathrm{amb}}-\dot{\mathbf{m}}_{\text {out }} \mathbf{T}\right)-p \frac{d \mathbf{V}}{d t}\right]
$$

where $\mathbf{T}$ is a vector containing the temperatures of all the elements, the lifting gas and the ballonet air, $\left[c_{v} m\right]$ is a diagonal matrix of the specific heat capacity (at constant volume for the lifting gas and ballonet air) for each element multiplied by the element's mass, $\dot{\mathbf{Q}}$ is the total heat flux for each element and for the gases, $\dot{\mathbf{m}}_{\text {in }}$ and $\dot{\mathbf{m}}_{\text {out }}$ are vectors containing the mass flow for each element, which is zero for all elements except for the ballonet air, $p$ is the pressure in the airship which is the same for the lifting gas and the ballonet air and $\mathrm{d} \mathbf{V} / \mathrm{d} t$ is the rate of change of volume of each element. Equation (36) is solved numerically using the implicit Euler method as shown in equation (37).

$$
\begin{aligned}
\mathbf{T}_{t+\Delta t}= & \mathbf{T}_{t}+\left[c_{v} m\right]^{-1}\left[\dot{\mathbf{Q}}\left(\mathbf{T}_{t+\Delta t}\right) \Delta t\right. \\
& +R\left(\dot{\mathbf{m}}_{\mathrm{in}} T_{\mathrm{amb}}-\dot{\mathbf{m}}_{\mathrm{out}} \mathbf{T}_{t+\Delta t}\right) \Delta t \\
& -p \Delta \mathbf{V}]
\end{aligned}
$$

The subscript $t$ denotes a value at the current timestep and $t+\Delta t$ at the next timestep. The implicit method is used because it is unconditionally stable. It can be seen that the new temperature values are used to calculate the heat fluxes, as such there is no closed form solution to equation (37). Instead the new temperature must be found numerically; in this simulation a Newton-Krylov solver is used. The change in volume for the lifting gas and ballonet, $\Delta \mathbf{V}$ in equation (37), can be found from the ideal gas equation. For the lifting gas:

$$
\frac{p_{g, t} V_{g, t}}{T_{g, t}}=\frac{p_{g, t+\Delta t} V_{g, t+\Delta t}}{T_{g, t+\Delta t}}
$$


and for the ballonet:

$$
\frac{p_{b, t} V_{b, t}}{T_{b, t} m_{b, t}}=\frac{p_{b, t+\Delta t} V_{b, t+\Delta t}}{T_{b, t+\Delta t} m_{b, t+\Delta t}}
$$

By noting that the pressures are the same in each gas, that is $p_{g, t}=p_{b, t}=p_{t} \quad$ and $\quad p_{g, t+\Delta t}=p_{b, t+\Delta t}=p_{t+\Delta t}$ equations (38) and (39) can be equated and manipulated to remove the pressure terms:

$$
\frac{V_{g, t+\Delta t}}{V_{b, t+\Delta t}}=\frac{V_{g, t} T_{g, t+\Delta t} T_{b, t} m_{b, t}}{V_{b, t} T_{g, t} T_{b, t+\Delta t} m_{b, t+\Delta t}}=K
$$

By noting that both the lifting gas and ballonet must experience an equal but opposite volume change:

$$
\begin{gathered}
\frac{V_{g, t+\Delta t}}{V_{b, t+\Delta t}}=\frac{V_{g, t}+\Delta V}{V_{b, t}-\Delta V}=K \\
\Rightarrow \Delta V=\frac{K V_{b, t}-V_{g, t}}{1+K}
\end{gathered}
$$

\subsubsection{Boundary Constraints}

If the volume of the lifting gas increases enough, the ballonet will empty and the membrane will rest along the base of the airship. The transition point from when there is air in the ballonet to no air can be found from the mass flow rate for the current timestep and the mass in the ballonet at the beginning of the timestep:

$$
\Delta \mathrm{t}_{\mathrm{m}}=\frac{m_{b}}{\dot{m}}
$$

If $\Delta t_{m}<\Delta t$ then the ballonet will empty in the current timestep and so the current timestep must be split into two parts. The first will continue with the method described above using the timestep $\Delta t_{m}$. Because the new ballonet mass will be zero, equation (41) cannot be used to find the change in volume. However, as the lifting gas will be fully expanded in the airship at the end of this timestep, the volume change will be the difference between the lifting gas' current volume and the airship volume:

$$
\Delta V=V_{A}-V_{g, t}
$$

where $V_{A}$ is the airship volume. The new pressure of the lifting gas can then be found using equation (38). Then the simulation will move to a constant volume model in which the lifting gas is fully expanded in the airship and so there is no pressure-volume or flow work:

$$
\mathbf{T}_{t+\Delta t}=\mathbf{T}_{t}+\left[c_{v} m\right]^{-1} \dot{\mathbf{Q}}\left(\mathbf{T}_{t+\Delta t}\right) \Delta t
$$

where $\dot{Q}_{b}=0$. The new pressure can be found from a simplification of equation (38):

$$
p_{g, t+\Delta t}=p_{g, t} \frac{T_{g, t+\Delta t}}{T_{g, t}}
$$

The simulation will continue to use the constant volume model until the lifting gas pressure falls below the critical pressure, that is the external ambient pressure plus the required super-pressure. When this happens, the previous timestep will need to be repeated. First, the time until the pressure is equal to the critical pressure will be found. Unlike the case for equation (42), there is no direct relation between pressure and time so the bisection method is used to determine $\Delta t_{p}$, the time increment at which the lifting gas pressure is within $0.01 \mathrm{~Pa}$ of the critical pressure. At this point the ballonet is empty and the pressure in the lifting gas is equal to the critical pressure and about to decrease. The instantaneous mass-flow into the ballonet at this point can be found from equation (34). This will be very large, as there is no pressure in the ballonet. As the ballonet fills with air this mass-flow rate will rapidly decrease. Care must be taken therefore to use a small time increment for this first timestep or there will be a large error caused by integrating this large mass flow rate over a large time difference. The gas conditions for the ballonet air and lifting gas at the end of the timestep can be determined by making some assumptions about the ballonet air: that its temperature is equal to the ambient atmospheric temperature and that it will have a mass of $\dot{m} \Delta t$ at the end of the timestep.

The gas in the ballonet can be modelled with the ideal gas law:

$$
p_{t+\Delta t}=\frac{(\dot{m} \Delta t) R_{\mathrm{air}} T_{\mathrm{amb}}}{\Delta V}
$$

and the lifting gas must follow Boyle's law:

$$
p_{t+\Delta t}=p_{t} \frac{V_{t} T_{t+\Delta t}}{V_{t+\Delta t} T_{t}}
$$

Equating the pressures of the lifting gas and ballonet and rearranging gives

$$
\frac{V_{t}-\Delta V}{V_{t} \Delta V}=\frac{T_{t+\Delta t} p_{t}}{T_{t}(\dot{m} \Delta t) R_{\mathrm{air}} T_{\mathrm{amb}}}=K_{2}
$$

so re-arranging for $\Delta V$ gives

$$
\Delta V=\frac{V_{t}}{K_{2} V_{t}+1}
$$

Then the lifting gas and element temperatures can be found from

$$
\mathbf{T}_{t+\Delta t}=\mathbf{T}_{t}+\left[c_{v} m\right]^{-1}\left(\dot{\mathbf{Q}}\left(\mathbf{T}_{t+\Delta t}\right) \Delta t-p \Delta \mathbf{V}\right)
$$

and the ballonet air temperature is equal to the ambient air temperature. Following on from this equation (37) can then be used as the ballonet fills back up with air.

\section{MODEL VALIDATION}

The model is validated against experimental results from Harada et al. (2003) [23]. The experimental apparatus was an airship that had an affixed solar array and 10 internal gas chambers separated by membranes as shown in Fig. 3. The 
five upper chambers contain the helium, the lower chambers contain air. To apply the thermal model to this structure, the membranes between each vertical set of helium and air chambers is assumed to be flexible so that volume changes can occur but the membranes between adjacent chambers is assumed to be rigid. Temperature measurements were taken on the envelope and solar array surfaces and at various points inside the gas chambers. The air temperature, wind speed and solar and IR radiation was also recorded. These inputs and the results from the experiment produced graphs in the paper that were vectorised and sampled for the validation using the WebPlotDigitizer tool [24]. The sampled data was linearly interpolated to provide inputs for every time step of the simulation.

The direct solar radiation, ground and sky infra-red radiation, air temperature and wind speed inputs are all taken from the published results. Diffuse and reflected solar radiation was calculated using SMARTS2 rather than data from Harada because these are very low and could not be extracted from the published graphs. In addition, because these contributions are two orders of magnitude smaller than the direct solar radiation, any errors in their calculation are expected to have a minimal effect on the validation. The solar angle was also calculated using SMARTS2.
Fig. 44 shows the validation results. Although the experimental data from Harada starts at 8 am local time, the comparison here is only from 9 am. The first hour is discounted as the results of the model are influenced by the guessed initial temperatures during this first hour. Fig. 4 also shows the results of previous models that have validated against the data from Harada. Table 1 shows the statistical measures of fit, $r$, and the root mean squared deviation (RMSD), from the model compared to the experimental data.

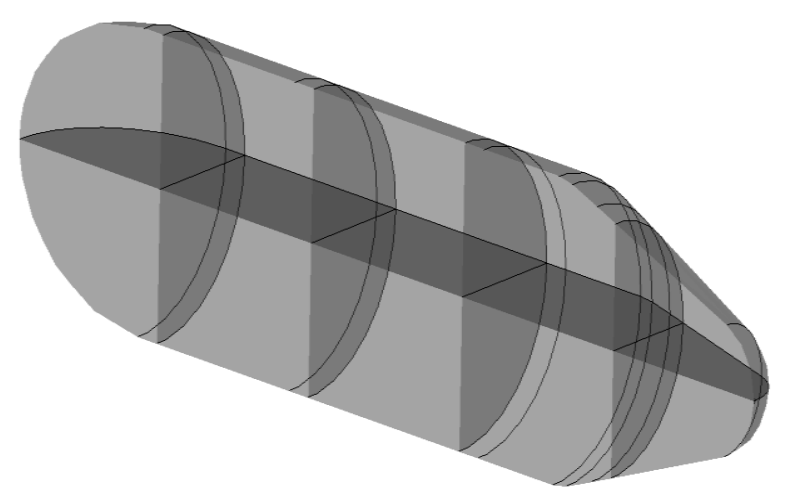

Fig. 3. Cross-section of the model of the Harada airship
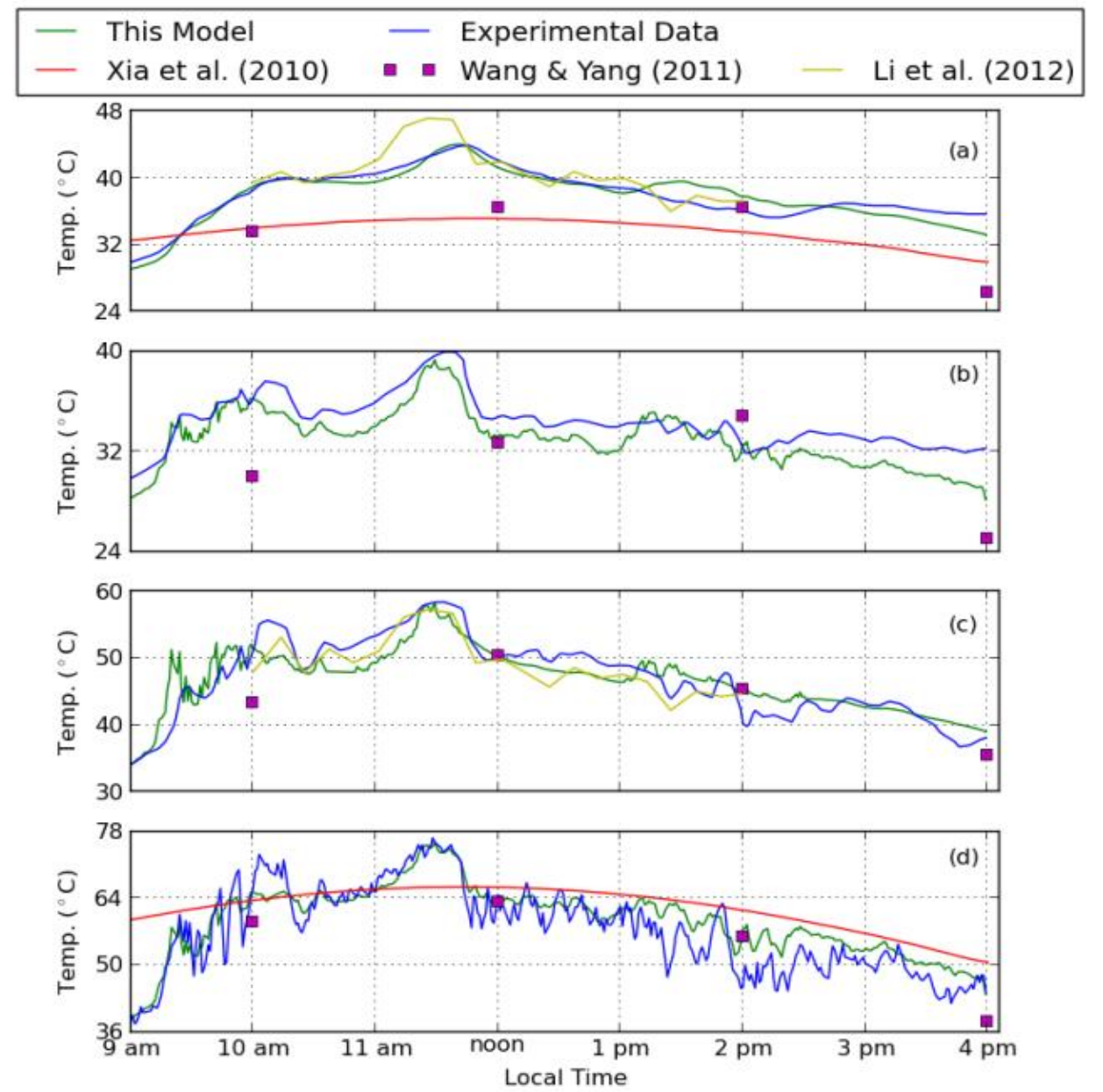

Fig. 4. Comparison of the (a) mean helium, (b) mean envelope, (c) maximum envelope and (d) maximum solar array temperatures of this model with the experimental data from Harada (2003) along with previous validation attempts by Xia et al. [10], Wang and Yang [13] and Li et al. [14] 
Table 1: Statistical measures of fit for validation

\begin{tabular}{|l|l|l|}
\hline & $r$ & $\operatorname{RMSD}\left({ }^{\circ} \mathrm{C}\right)$ \\
\hline Mean Helium & 0.934 & 1.12 \\
\hline Mean Envelope & 0.914 & 1.63 \\
\hline Max Envelope & 0.900 & 2.70 \\
\hline Max Solar Array & 0.914 & 4.26 \\
\hline
\end{tabular}

The results of the validation are encouraging. A correlation coefficient of 0.9 or above for each case indicates that the model follows the trend of the experimental results well and the RMSD values indicate that the model temperatures are close to the experimental results, although it is clear that the helium temperatures are modelled better than the solar array temperatures. There are 420 data points for each time-series so the correlation has a high significance. From a visual inspection of Fig. 4 it can be seen that this model is an improvement over previous models, only the maximum envelope temperature modelled by Li et al. (2012) is as close to the experimental data as this model.

\section{CONCLUSION}

Thermal analysis of airship, aerostat and scientific balloon designs aids in the assessment of their feasibility. In this paper, a thermal model was developed for an airship that included a ballonet used to control the pressure of the lifting gas. The validity of this new model was demonstrated and shows a good agreement to previously published experimental data. The new thermal model presented in this paper shows a stronger agreement with the experimental data than previously published models and as such can be used to provide a better analysis of future aerostat or airship designs that incorporate ballonet structures, such as the power-raising aerostat design proposed by Aglietti et al.

\section{REFERENCES}

[1] G. S. Aglietti, T. Markvart, A. R. Tatnall, and S. J. Walker, "Solar Power Generation Using High Altitude Platforms Feasibility and Viability," Prog. Photovolt: Res. Appl, vol. 16, pp. 349-359, 2008.

[2] G. S. Aglietti, T. Markvart, A. R. Tatnall, and S. J. Walker, "Aerostat for electrical power generation concept feasibility," Proceedings of the Institution of Mechanical Engineers, Part G: Journal of Aerospace Engineering, vol. 222, pp. 29-39, 2008.

[3] G. S. Aglietti, S. Redi, A. R. Tatnall, and T. Markvart, "High Altitude Electrical Power Generation," WSEAS Transactions On Environment And Development, vol. 4, no. 12, pp. 1067-1077, 2008.

[4] G. S. Aglietti, S. Redi, A. R. Tatnall, and T. Markvart, "Harnessing High-Altitude Solar Power," IEEE Transactions On Energy Conversion, vol. 24, no. 2, pp. 442-451, 2009.

[5] S. Redi, G. S. Aglietti, A. R. Tatnall, and T. Markvart, "An Evaluation of a High Altitude Solar Radiation Platform," Journal of Solar Energy Engineering, vol. 132, 2010.

[6] S. Redi, G. S. Aglietti, A. R. Tatnall, and T. Markvart, "Dynamic Response to Turbulence of Tethered
Lighter-Than-Air Platforms," Journal of Aircraft, vol. 48, no. 2, pp. 540-552, 2011.

[7] A. Morris, ed., Scientific Ballooning Handbook. NCAR TN/1A-99, Boulder, CO: National Center for Atmospheric Research, 1975.

[8] Leland A Carlson and WJ Horn, "New thermal and trajectory model for high-altitude balloons," Journal of Aircraft, vol. 20, no. 6, pp. 500-507, 1983.

[9] Rodger E. Farley, "BalloonAscent: 3-D simulation tool for the ascent and float of high-altitude balloons," 2005. AIAA.

[10] Xin-Lin Xia, De-Fu Li, Chuang Sun, and Li-Ming Ruan, "Transient thermal behavior of stratospheric balloons at float conditions," Advances in Space Research, vol. 46, no. 9, pp. 1184-1190, 2010.

[11] Qiuming Dai, Xiande Fang, Xioajian Li, and Lili Tian, "Performance simulation of high altitude scientific balloons," Advances in Space Research, vol. 49, pp. 1045-1052, 2012.

[12] R. M. Rapert, "A heat transfer model to a heated helium airship," Master's thesis, Naval Postgraduate School, 1987.

[13] YW Wang and CX Yang, "A comprehensive numerical model examining the thermal performance of airships," Advances in Space Research, vol. 48, pp. 1515-1522, 2011.

[14] Xiaojian Li, Xiande Fang, Qiuming Dai, and Zhanru Zhou, "Modeling and analysis of floating performances of stratospheric semi-rigid airships," Advances in Space Research, vol. 50, no. 7, pp. 881890, 2012.

[15] Qiumin Dai, Xiande Fang, and Yu Xu, "Numerical study of forced convective heat transfer around a spherical aerostat," Advances in Space Research, vol. 52, no. 12, pp. 2199-2203, 2013.

[16] Xioajian Li, Xiande Fang, and Qiuming Dai, "Research on Thermal Characteristics of Photovoltiac Array of Stratospheric Airship," Journal of Aircraft , vol. 48, no. 4, pp. 1380-1386, 2011.

[17] Yiran Wang and Yunxia Liu, "Numerical Simulation about Thermal Environment of Solar Energy Airship in Stratosphere," Procedia Engineering, vol. 29, pp. 1745-1749, 2012.

[18] C. Gueymard, "SMARTS, A Simple Model of the Atmospheric Radiative Transfer of Sunshine: Algorithms and Performance Assessment.," Professional Paper FSEC-PF-270-95, Florida Solar Energy Center, 1679 Clearlake Rd., Cocoa, FL 32922., 1995.

[19] Oleg A. Louchev, "Steady state model for the thermal regimes of shells of airships and hot air balloons," Int. J. Heat Mass Transfer, vol. 35, no. 10, pp. 2683-2693, 1992.

[20] Jin, Menglin, and Shunlin Liang. "An improved land surface emissivity parameter for land surface models using global remote sensing observations." Journal of Climate vol. 19, no. 12 pp 2867-2881, 2006.

[21] George N. Walton, "Calculation of Obstructed View Factors by Adaptive Integration,” Tech. Rep. NISTIR 6925, NIST, Nov 2002. 
[22] Qiumin Dai, Xiande Fang, and Xiaojian Li, "Dynamic simulation of breakaway characteristics of tethered aerostats," Advances in Space Research, vol. 48, no. 7, pp. 1258-1264, 2011.

[23] Kenya Harada, Kunihisa Eguchi, Masaaki Sano, and Shuichi Sasa, "Experimental study of thermal modeling for stratospheric platform airships," AIAA, vol. 6833, 2003.

[24] "WebPlotDigitizer." arohatgi.info/WebPlotDigitizer Accessed on 2014-07-01. 